\title{
IMPLANTACIÓN DEL PROYECTO DE INSERCIÓN DEL ACOMPAÑANTE EN EL PARTO: EXPERIENCIAS DE LOS PROFESIONALES
}

\author{
IM PLANTING AN IN SERTION PROJECT CONCERNING SIGNIFICANT \\ FAM ILY COM PANY DURING LABOR: PROFESSIONAL EXPERIENCES
}

CLEUSA MAIA DE SOUZA PINTO* y LUIZA AKIKO KOMURA HOGA**

\begin{abstract}
RESUMEN
Esta investigación tuvo el objetivo de describir las experiencias de los profesionales en la implantación del proyecto de inserción del acompañante en el parto. M étodo: El estudio fue realizado en el centro de parto normal de un hospital vinculado a la Secretaría de Estado de la Salud de São Paulo. El abordaje de investigación fue la cualitativa y los datos fueron recolectados por medio de la observación del escenario y entrevistas con 24 profesionales, entre ellos médico obstetra y neonatólogo, enfermera obstetra y auxiliar de enfermería. Resultados: Las categorías descriptivas dela experiencia fueron: a) Los profesional es tuvieron que superar muchas creencias y val ores negativos en relación a los acompañantes durante el parto; b) Los profesional es no tenían experiencias con acompañantes y el inicio de la asistencia al parto con la presencia de ellos causó mucha ansiedad. Conclusiones: La implantación de un proyecto de inserción del acompañante en el parto requiere preparación previa del equipo multiprofesional. Esta preparación puede evitar desgastes innecesarios de los profesionales y contribuir en el éxito del proyecto.
\end{abstract}

Palabras claves: Parto, equipo de asistencia al paciente, centro de parto normal.

\begin{abstract}
Theaim of this research wasto describetheprofessional 's experiences related to theinsertion of companion in childbirth. M ethod: This study was carried out at the birth center of an institution connected to the São Paulo State Government. The research approach was qualitativeand the data was collected through the observation of thescenery and interviews with 24 professionals, members of the professional staff including obstetric and neonatal physicians, nurse midwifes and nursing aids, as well. Results: The descriptive categories of the experience were: a) Professionals have overcome many negative beliefs and values related to the childbirth companion; b) Lack of experience has provoked anxiety during the beginning of the project of insertion of companion in childbirth. Conclusion: Professional staff need to be adequately prepared early to the implementation of a project of insertion of companion in childbirth. This previous preparation can avoid unnecessary professional stress and to promote the success of the project.
\end{abstract}

Keywords: Childbirth, professional staff, birth center.

Recepcionado: 17.03.2004. Aceptado: 13.05.2004

\section{INTRODUCCIÓN}

La humanización dela asistencia del parto ha sido el blanco de la atención de profesionales y personas, directa o indirectamente, involucradas con la práctica obstétrica. Ella abarca la cuestión del acompañanteque, actual mente, se encuentra aún excluido del proceso del parto en muchas instituciones hospitalarias del contexto brasileño. El respeto a la libertad de elección del acompañante durante el trabajo de parto por la parturienta y el monitoreo adecuado de su bienestar físico y emocional son comprobadamente útiles y deben ser estimuladas (OMS, 1996).

Esta prerrogativa está asegurada legalmen-

\footnotetext{
*Enfermera obstérica, Hospital General de Itapecería da Serra, Sao Paulo. Brasil.

**Enfermera obstétrica. Livre docente en enfermería. Escuela de Enfermería de la Universidad de Sao Paulo. Sao Paulo, Brasil.E-mail: kikatuca@usp.br
} 
te en el ámbito del Estado de São Paulo-Brasil, desde 1999, por Ley no 10.241 (São Paulo, 1999). Ella determina, entre otros aspectos, el respeto al derecho de la parturienta de tener un acompañante desu elección en la hora del parto. A pesar de queella ya está en vigor hace algún tiempo, su cumplimiento está siendo tratado con negligencia por varias instituciones.

El Ministerio de Salud del Brasil (Brasil, 2000; Brasil, 2001) también estableció un protocolo asistencial indicando la necesidad de que la parturienta disponga de un acompañante de su elección, caso éste sea su deseo. Esta prerrogativa aún no está incorporada en la práctica de los profesionales ni en las normas y rutinas de muchas instituciones.

Por tratarse de una práctica innovadora para gran parte de las instituciones, se cree que su implantación demanda revisión de conceptos de asistencia y reflexión acerca de los prejuicios que la involucran. Son necesidades que merecen ser atendidas, sin embargo requieren cambios deactitudes de muchos profesionales que poseen creencias y valores profundamente arraigados que provocan el rechazo de la idea de la presencia del acompañante en el parto.

Se evalúa que la superación de los prejuicios y de las creencias y valores que perjudican la implantación de prácticas benéficas, para el proceso de nacimiento y parto, sean etapas fundamentales para una asistencia obstétrica adecuada desde el punto de vista técnico y humanístico (Brasil, 2001; OM S, 1996).

Investigaciones ya comprobaron que el apoyo físico, empático y continuo durante el trabajo de parto beneficia a las parturientas. El trabajo departo setorna más breve, las medicaciones y analgesias son utilizadas con menor frecuencia, los recién nacidos presentan menos escores de Apgar abajo de 7 y la necesidad del parto operatorio es menor (Klausy Kennel,1993; Enkin, Keirse y Renfrew, 1989; Hemminki, Virtay Koponen, 1990; H ofmeryr, Nikodem, Wolman, Chalmers y Kramer (1991).
Son evidencias científicas que contribuyeron para invertir la práctica predominanteen la década de los 60, cuando no era necesaria la presencia del padre en el nacimiento. La tendencia actual es su presencia en el parto y las principales razonesqueproporcionaron tal cambio fueron las transformaciones ocurridas en las posturas profesionales y el propio deseo de los padres de participar en el proceso (Vehvilãinem-Julkunen; Linkoonen, 1998).

A pesar que los profesionales presenten tendencia a aceptar la presencia del padre en el parto, esta práctica aún está envuelta por sentimientos de aprehensión. Profesionales quetrabajaron con hombres en el proceso del nacimiento refirieron sentimientos de aprehensión, ansiedad en el desarrollo de estetipo depráctica. Son experiencias que provocaron aumento en la demanda de trabajo del equipo de salud y algunos fracasos fueron factores que contribuyeron a mantener al hombre apartado del proceso (Brown, 1982).

Ofrecer apoyo empático y continuo durante el trabajo de parto es comprobadamente útil y debe ser proporcionado a las parturientas. Es un trabajo que puede ser plenamente desempeñado por los acompañantes. Se preconiza que las instituciones que prestan asistencia al parto deben permitir la presencia del acompañanteescogido por la mujer en la asistencia al parto, de forma sistematizada (Brasil, 2001).

Se estima, entretanto, quela implantación de un proyecto de inserción del acompañanteen el parto requiere preparación previa por parte de los profesionales. La descripción sistematizada de experiencias de otras instituciones puede servir como punto de partida en la elaboración de programas destinados a la capacitación de estos profesionales. Es un trabajo que puede evitar desgastes innecesarios de los miembros del equipo multiprofesional, el fracaso del proyecto y su consecuente interrupción.

La descripción de experiencias relativas a la implantación de proyectos deinserción del acompañante en el parto no fueron encon- 
trados en la literatura, por lo que ésta fue la justificación para realizar esta investigación.

Este estudio tuvo como objetivo describir las experiencias de los profesionales en la implantación del proyecto deinserción del acompañante en el parto.

\section{METODOLOGÍA}

\section{Tipo de estudio}

Se trata de una investigación desarrollada en el paradigma cualitativo. Éste hace posible la comprensión de los valores y representaciones de determinado grupo en relación a temas específicos y el conocimiento de los significados, actitudes y hábitos involucrados (M inayo, 1997). El desarrollo de la investigación qualitativa tieneel objetivo de comprender el contexto del cuidado y utilizar este conocimiento para promover la salud delas personas. Para queesto suceda es esencial quelas experiencias humanas sean muy bien comprendidas (Hinds; Chaves y Cypess, 1992).

\section{Local}

La investigación fue desarrollada en el Centro de Parto Normal (CPN) del Hospital General de Itapecerica da Serra (HGIS), M unicipio localizado en el Estado de São Paulo, Brasil. En esa institución la presencia del acompañantedeelección dela parturienta fue incluida como partedel protocolo deasistencia. Este fue el factor determinante de la elección de esta institución para la realización de la presente investigación. Ella está vinculada a la Secretaría del Estado de Salud y cuenta con 169 camas, de las cuales 81 son destinadosala prestación deasistencia en ginecología y obstetricia y atiende a cerca de 450 parturientas/mes. El proyecto de investigación fue presentado a la dirección del hospital, quien autorizó la ejecución de la investigación.

\section{Población del estudio}

La asistencia obstétrica y neonatal en el H GIS es prestada por el equipo multidisciplinario compuesto por médico obstetra y neonatólogo, enfermera obstétrica, nutricionista, asistente social, psicóloga, técnicos y auxiliares de enfermería. Fueron solicitadosa colaborar en la investigación sólo los profesionales que tenían contacto directo con la parturienta y su acompañante.

Colaboraron en este estudio 24 profesionales, de los cuales seis médicos obstetras (M O), seis médicos neonatólogos ( $M N$ ), seis enfermeras obstétricas (EO), tres técnicas de enfermería (TE) y tres auxiliares de enfermería (AE). Los criterios de inclusión de los colaboradores fueron haber actuado por los menosun año en la institución y demostrado disponibilidad para participar.

\section{Recolección de datos}

El proyecto de investigación fue previamente aprobado por la Comisión de Ética e Investigación de la institución. Fueron obedecidos los criterios que constan en la Resolución no 196/96 que trata de las Directrices Éticas y Normas que Reglamentan la Investigación en Seres Humanos (Brasil, 1996).

Los datos fueron recolectados por medio deobservaciones del escenario donde se presta la asistencia a la parturienta y entrevistas. El contexto de ocurrencia del cuidado debe ser observado porque él es una fuente rica de datos, posee un significado y debe ser comprendido (Hinds y col., 1992). En esta investigación la observación del escenario se restringió a las actividades desempeñadas por los profesionales y a la planta física dondesepresta la asistencia durante el trabajo de pre-parto y parto.

Las entrevistas fueron conducidas por medio de preguntas no estructuradas de acuerdo 
con Kvale (1996). La pregunta inicial realizada a los profesionales al inicio de las entrevistas fue: Describa ¿cómo fuesu experiencia en la fase de implantación del proyecto deinserción del acompañante en el parto?

Las entrevistasfueron previamenteprogramadas en horario y local de preferencia de cada colaborador. Antes del inicio, ellos fueron nuevamente aclarados sobre la finalidad de la investigación y se les solicitó firmar el consentimiento informado, en el cual constaba que ellos consentían la grabación de las entrevistas y la utilización de su contenido para fines científicos, además de la garantía dada sobre la obediencia de los aspectos éticos referidos en la citada resolución. Las entrevistas duraron entre 30 y 60 minutosy fue ron realizadas entre los meses de mayo y junio del 2001. Todos prefirieron ser entrevistados en la propia institución, en el horario de trabajo que permitía el desarrollo de este tipo de investigación, sin perjuicios para la asistencia.

No fue establecido anticipadamenteel número de colaboradores, sin embargo la inclusión de ellos fue encerrada cuando se observó la constante repetición de experiencias, lo queen la investigación cual itativa se denomina saturación teórica y constituye el parámetro derigor en el abordajemetodológico adoptado (M orse, 1998).

\section{Análisis de los datos}

Las entrevistas fueron sometidas al proceso detranscripción inmediatamente después de su término, conforme el preconizado por M eihy (1996). Éstesugierela transcripción de las entrevistas grabadas en forma escrita. En esta primera fase, se realizan las correcciones de los vicios de lenguaje y de los errores gramaticales y suprimen las repeticiones excesivas. Su finalidad es la presentación de un texto claro, con mantención del sentido intencional dado por el entrevistado. La etapa a seguir es la textualización, realizada para dar lógica al texto y suprimir las preguntas. En la tercera fase, de transcripción, el investigador recrea el texto con el objetivo final de clarificar las narrativas.

Cada narrativa transcreada fue enviada al colaborador correspondiente. Ellos recibieron el íntegro de sus narrativas e hicieron las correcciones deseadas. Éstas fueron totalmente incorporadas resultando en la narrativa final, aprobada por los respectivos colaboradores de la investigación.

El análisis cualitativo involucra el método inductivo o el proceso de elaboración degeneralizaciones a partir de los datos. En este trabajo, el investigador debe permanecer atento a los temas que surgen y al modo de categorizarlos. Esta etapa requiere queel investigador mantenga gran sensi bilidad y apertura en relación al conjunto de datos, sin valorizar algunos puntos o menospreciar otros. El análisis cualitativo desarrollado de esa forma permite la producción de "insights" profundos en una visión holística del fenómeno (Polit y Hungler, 1995; Janesick, 1998).

Con base en estos presupuestos, fue desarrollado el análisis de los datos, conforme la proposición de Janesick (1998). Esta autora compara el "arte" de analizar datos cualitativos con la construcción de una coreografía, en este caso, las categorías descriptivas de las experiencias de los profesionales relativas a la fase de implantación del proyecto de inserción del acompañante en el parto. Ellas deben retratar de forma realista la experiencia vivida, demodo tal queel lector dela investigación pueda comprender el universo interrelacionado de los datos.

\section{RESULTADOS}

Primeramente, se presentan las principales características de la asistencia prestada en la institución y categorías descriptivas de la experiencia. Sus partes constituyentes son ejemplarizadas por medio de pequeños trechos extraídos de los discursos, cuyas fuentes son 
identificadas por la mención abreviada de la categoría profesional, mencionada anteriormente.

\section{Características de la asistencia prestada en la institución}

La asistencia prestada, norteada por principios humanísticos y la presencia del acompañante el egido por la parturienta, está institucionalizada. La institución está acreditada como Hospital Amigo del Niño y en el año 2000 recibió el Premio Nacional Prof. Galba de Araújo, implantado por el Ministerio de Salud para estimular el uso de tecnología apropiada para el parto y la atención humanizada (Brasil, 2000).

El ambiente físico destinado a la atención de las parturientas está situado fuera del área quirúrgica, siendo encaminados al Centro Quirúrgico Obstétrico (CCO) (apenas) los partos que no son normales. El modelo de asistencia del parto adoptado en la institución es considerado uno de los pioneros en el ámbito nacional y sigue las recomendaciones de la Organización M undial dela Salud en lo que serefierea la atención del parto y nacimiento (OM S, 1996). Cabe resal tar que en esta institución la mujer recibe asistencia durante el trabajo de parto, parto y post parto inmediato en el mismo ambiente físico, denominado Sistema PPP. La presencia del acompañante elegido por la parturienta es permitida y estimulada. Él recibeorientaciones del equipo de enfemería respecto a las actividades que pue de desempeñar en el trabajo de pre-parto y parto.

\section{Las categorías descriptivas de la experiencia}

a) Los profesionales tuvieron que superar muchas creencias y valores negativos en relación a los acompañantes

Muchos profesionales refirieron creencias y valores negativos en relación a los acompa- ñantes. Ellos estaban relacionados a la incertidumbre en cuanto a los beneficios que los acompañantes podrían proporcionar y preocupaciones relativas a posibles perjuicios provocados por la presencia del acompañante. Existía el temor de que los acompañantes no siguieran correctamente las orientaciones dadas sobre el papel a desempeñar para ayudar en la asistencia al parto. Los profesionales tenían también preocupación en cuanto a una posible interferencia de las acciones desempeñadas por los acompañantes en relación a la evolución del trabajo de pre-parto y parto. Temían también queellos cuestionen las conductas médicas adoptadas en el transcurso de la asistencia al parto.

Hallaba queél no iba a seguir las orientaciones y que iba a obstaculizar (AE 3). Yo me preocupaba con el hecho de que ellos hagan algo que perjudicara el parto (MO 4); temía que ellos quedasen cuestionando nuestras conductas, dudando de nuestra capacidad (MO 5, EO 2).

Muchos acreditaban también que ellos podrían obstaculizar dealguna forma la rutina de la asistencia o perturbar la privacidad de otras mujeres y hasta colocar en riesgo algún aspecto de la asistencia.

Imaginaba que ellos podrían perturbar la rutina (AE 2). La presencia de ellos podría quitar la libertad de las otras mujeres (AE 4). De repente, ellos podían hacer algo que pongan en riesgo la vida de la mujer y del niño (M N 3)

Con base en tales experiencias, los profesionales evaluaron que un proyecto de implantación relativa a la inserción del acompañante en la asistencia al parto debe ser precedida por un trabajo quetenga como objetivo superar prejuicios y el imaginario negativo en relación al acompañante que pudiese perjudicar nuevas iniciativas con propuesta semejante.

Lo que dificulta es la postura del profesional de salud que tiene recelo, prejuicios, piensa que él va a obstaculizar... todo eso precisa ser superado (EO 4; EO 5) 
Los profesionales evaluaron también que hay necesidad de que la idea positiva, en relación al acompañante, debe estar incorporada en el conjunto de profesionales. La asistencia al parto es desarrollada por un equipo, lo que requiere preparación y educación continuada de todos los profesionales involucrados directa o indirectamente en su asistencia.

Resaltaron también que es prudente evitar proponer un proyecto de inserción del acompañanteen el parto deforma impositiva. Consideraron queestetipo de propuesta debe ser preliminarmente trabajada de forma de mocrática y participativa, con el objetivo principal de hacer que el conjunto de profesionales pasen a tener convicción en relación a la propuesta, pues ésta es una etapa fundamental para la obtención del éxito en el proceso.

No adelanta en nada tratar de imponer al profesional la idea del acompañante. Todo el equipo debe ser primero trabajado, preparado, convencido para tener un objetivo común (MO 1 , EO 1, 2, 5, 6; AE 2, TE 2,3).

b) Los profesionalesno tenían experiencias con acompañantes y el inicio de la asistencia al parto con su presencia causó mucha ansiedad

Losprofesionalesfueron unánimesen afirmar queellosno tenían experiencias anteriorescon acompañantesen el parto. No tuvieron estetipo de oportunidad durantela fase de formación profesional en los cursos de pregrado. Contenidos relativos a la importancia que el acompañante tiene para las parturientas o los beneficios que proporciona al vínculo familiar no hacían parte de los currículos de los cursos del pregrado.

Aún después del pregrado algunos profesionales no tuvieron la oportunidad de trabajar con acompañantes en el parto, porque las instituciones donde habían actuado hasta ese entonces no permitían la presencia del acompañante en el parto.
No tuve experiencia con acompañante durante las prácticas en la época del pregrado. Ese tipo de asunto no era abordado en las escuelas (EO 3). Trabajé 18 años sólo con el modelo tradicional, sin acompañante (M O 2).

Cuando el protocolo asistencial con la prerrogativa dela presencia del acompañantefue implantado, hubo un período de transición queduró mucho tiempo. En esteperíodo ellos intentaron familiarizarse con la nueva práctica, fase en quetuvieron que mantener constante preocupación en diversos ámbitos de la asistencia. Ellos dudaban de la capacidad de los acompañantes para seguir correctamente las orientaciones recibidas. Temían los problemas que ellos pudiesen provocar en el transcurso de la asistencia, como, por ejemplo, la falta de higiene o una conducta intempestiva en cualquier momento, que podrían perjudicar a la parturienta o al recién nacido.

Como consecuencia, las primeras experiencias de los profesionales fueron acompañadas por la sensación deansiedad. Tal vivencia estaba relacionada sobre todo con el temor relativo a posibles ocurrencias negativas quepodrían surgir y que pudiesen perjudicar la atención a la parturienta y al recién nacido.

En el inicio, era una cosa teórica, hallaban que el acompañante iba a desmayarse; que no iba a entender el proceso del parto, que interferiría pidiendo cesárea, que quedarían asustados; me quedaba preocupado con todo, cualquier problema que pudiese acontecer, porque nosotros somos los responsables de la asistencia ( $M N$ 5; MO 4, TE 4)

El sentimiento deansiedad presentedurante todo el período de implantación y adaptación a un proyecto innovador fue un punto en común en la narrativa dela mayoría delos profesionales entrevistados.

Fue un período de gran ansiedad... M equedaba constantemente preocupado... vigilaba siempre que no aconteciera nada errado, porque nosotros somos los que respondemos legalmente por la asistencia... (MO 1,3;AE 3; TE 2, EO 1; M N 4) 
Algunos consiguieron adaptarsefácilmente al modelo. Otros, sin embargo, tuvieron dificultades, pero procuraron adecuarsea las propuestas del servicio para poder mantener su empleo. Hubo mención de que aquellos que no consiguieron ajustarse salieron de la institución, sea por voluntad propia o de la institución.

El período deadaptación fuedifícil, pero con el tiempo me ajusté a la propuesta de la institución (MO 4, MN 5, EO 1). Profesionales que no consiguieron adecuarse a las propuestas de asistencia de la institución fueron mandados a salir o salieron por propia voluntad (M 02)

Este sentimiento de aprehensión fue siendo superado a medida que en la práctica se indicaba lo contrario, o sea, que los acompañantes, en verdad, no generaban problemas. Al verificar que tales hechos se repetían, los argumentosy actitudes favorablesa la presencia del acompañante en el parto sefueron fortaleciendo. Esto hizo con que la sensación de inseguridad y temor en relación a los acompañantes fuesen sustituidos por la confianza en relación al trabajo desarrollado por ellos, puesto que pudieron constatar que era bené fico para la madre, el hijo y la familia.

Creía que los acompañantes obstaculizaban y esto me dejaba inseguro, aprehensivo en relación a ellos, hoy pienso lo contrario, ellos ayudan y mucho, en muchos aspectos, a la madre, al hijo ya la familia ( $\mathrm{M} O 3, \mathrm{MN} 2$ ). Con el tiempo pudeconstatar, con lasinnúmeras experiencias que fui teniendo, que el "yo" tenía como barrera. Hoy tengo como argumento incluir al acompañante en el parto, hoy no sabría trabajar sin él (MO 1, AE 6)

\section{DISCUSIÓN Y CONSIDERACIONES FINALES}

Esta investigación reveló que los profesionales poseen muchas creencias y val ores negativosen relación a la presencia del acompañante en el parto, hecho que generaba temor y desconfianza en relación a ellos. Sin embargo, las constataciones realizadas en el cotidiano del trabajo de que los acompañantes promueven vivencias positivas en el proceso del nacimiento y parto, fue determinante para revertir el imaginario negativo que los profesionales tenían en relación a los acompañantes. Esta transformación fue resultado de la adquisición de argumentos sólidos y dela confirmación, en la práctica, respecto a los beneficios proporcionados por los acompañantes. Son experiencias positivas que ayudan a promover la diseminación de la idea entre los pares de los profesionales.

El sentimiento de aprehensión en relación a la presencia del padre en el parto también fue observada en otra investigación (Brown, 1982). Seconcluye, por lo tanto, que propuestas nuevas de inserción del acompañante en el parto deben ser precedidas por profunda y amplia preparación de todos los profesionales involucrados.

Entrenamientos o reciclajes periódicos de estos profesionales también son necesarios. Se demostró que, en cuanto este trabajo no es realizado, hay posibilidad de que las actitudes de resistencia y alejamiento frente a ese tipo de propuesta persistan, sobre todo en razón del desconocimiento relativo a los beneficios que los acompañantes proporcionan a las parturientas, sus familias y a los propios miembros del equipo de salud (Brown, 1982).

Teniendo en consideración la existencia de evidencias científicas que comprueban la eficacia de la presencia del acompañante en el parto, evaluamos que la superación de los obstáculos iniciales enfrentados por los profesionales debe ser trabajada eincentivada. Se evalúa que ésta sea una forma de preservar el derecho básico y fundamental relativo a la obtención de asistencia al nacimiento y parto de buena calidad, que incluye la prerrogativa de la presencia del acompañante de elección de las parturientas.

Las posibilidades, beneficios, así como las dificultades relativas a la presencia del acompañante en el parto deben ser ampliamente difundidas en el medio profesional y en los 
medios de comunicación. El desconocimiento de estos aspectos puede estar perjudicando o retardando el proceso de inserción del acompañanteen el parto en muchosservicios de asistencia obstétrica. Sobretodo en el contexto brasileño, estetipo de propuesta deasistencia aún es incipiente, lo cual dificulta su divulgación. En consecuencia, tanto los estudiantes como los profesionales del área de la salud no tienen posibilidades amplias para adquirir este tipo de experiencia.

Consideramos que es esencial que la formación académica en el área de la salud y la propia asistencia acontezcan basadas en paradigmas claramente definidos. En ese sentido, servicios pioneros en términos de humanización dela asistencia obstétrica con inclusión del acompañanteen el parto, poseen gran importancia social en el contexto de asistencia obstétrica actual. Se cree que la inclusión dela presencia de personas en proceso deformación profesional, tal como ocurreen la institución donde esta investigación fue realizada, facilita la divulgación de esa práctica con bases más sólidas.

La vivencia preliminar de las experiencias directamentecon parturientas y susacompañantes es un factor determinante para la motivación y convicción de esos nuevos profesionales en la idea de la presencia del acompañante en el parto. Con esto, ellos estarán evitando eventuales contratiempos al inicio de la carrera. Además, podrán promover la implementación de nuevos proyectos, con adecuado fundamento teórico y práctico. La necesidad de integración del conjunto de profesionales directa 0 indirectamente con la asistencia fue mencionada. En su visión esta medida es relevante porque la sincronía de las acciones de los miembros del equipo esfundamental para el éxito del conjunto de acciones que son desarrollados en un proyecto.

Hay unanimidad en relación a la idea de queel acompañantees importantedado aque los eventuales recelos o falta de convicción pueden interferir negativamente en el proceso. El incentivo a la concretización práctica delas potencialidades de todos los profesionales es importante para promover el éxito final de cualquier propuesta de asistencia (Fabre, 1992).

Se considera también que la inclusión del acompañanteen la asistencia al parto debeser partedeun proyecto mayor, norteado por una filosofía de asistencia que abarque todos los aspectos relacionados a ella. Con eso se busca una asistencia de calidad que considere la eficiencia profesional yla satisfacción delasnecesidades en la perspectiva de las propias mujeres y respectivas familias. Por lo tanto, hay necesidad de obtener competencia técnica, disponer de habilidad y tener disponibilidad y dedicación para alcanzar el ideal que contempla tanto los aspectos técnicos como humanísticos del cuidado.

Losprofesionales involucrados con la educación y enseñanza representan figuras preponderantes en esta cuestión, sobretodo para la incorporación de la perspectiva humanística en la asistencia obstétrica. Se sugiere que las escuelas y los servicios de asistencia obstétrica preparen previamente a sus profesionales en este ámbito (Fustinoni, 2000; Gualda, 1993; Jeneral, 2000; O chiai, 2000; Silva, 1999, Zagonel, 1997). En ese sentido, se evalúa que la inclusión de ese tipo de experiencia en la fase de formación de los estudiantes de medicina, enfermería y profesionales afines sea una forma de concretizar la incorporación relativa a la validez y relevancia de un modelo de asistencia que incluya al acompañante. En la fase de formación son incorporados los princípios filosóficos de la profesión (Evans, 1999).

La transformación del cuadro actual de asistencia obstétrica, por lo menos en lo que serefiereal contexto brasileño, requierela incorporación denuevas actitudes por parte de los propios profesionales. Reestructuraciones en la filosofía de asistencia, para la inclusión de todos los profesionales y los múltiples aspectos relacionados a ella también son necesarias (Zagonel, 1997). Son transformaciones necesarias para promover el apoyo psicológi- 
co, el alivio del dolor y de la soledad en un momento crucial en la vida dela mujer (Enkin, Lumley y Keirse, 1995). Eso contribuiráa que la parturienta, su hijo y la familia vivan la experiencia del parto deforma saludableeintegradora y lleven consigo el recuerdo positivo de un momento intensamente vivido y permeado por importantes significados simbólicos.

\section{LIMITACIONESDE LA INVESTIGACIÓN}

Juzgamos que el hecho de que una de las investigadoras haga parte del equipo, que presta asistencia en la institución, demandó una constante actitud de extrañamiento en relación a los hechos observados, lo que posibilitó aprehenderlos de la forma más neutra posible. Fue necesario también admitir que el hecho de que una de las investigadoras sea miembro del equipo profesional puedehaber incrementado el deseo deobtener datosfavorables y positivos relativos a la presencia del acompañante. La conciencia de este hecho estuvo siempre en mente durante la realización de las entrevistas, observación del escenario estudiado y el análisis de los datos.

Por otro lado, es preciso resaltar que los profesionales tenían pleno conocimiento de los objetivos de la investigación y el hecho de saber queel servicio dondeactuaban era pionero podría ayudar al deseo de incrementar y potencializar experiencias positivas y menospreciar las negativas. Es necesario que el investigador cualitativo conviva con este peso, o sea, el de trabajar con datos plausibles 0 humanamente posibles y admitir las subjetividades individuales.

Los datos de este estudio, no obstante haber sido recolectados con miembros del equipo multidisciplinario y con inclusión de todas las categorías profesionales directamente involucradas en la asistencia, no permiten generalizaciones, pues se trata de un grupo distinto que tuvo preparación especializada.
Sus miembros, quieran o no, están inclinados a prestar este tipo de asistencia. En este sentido, se juzga pertinente desarrollar investigación con profesionales que no se adaptaron a la filosofía de asistencia y al servicio con la presencia del acompañante y que, en razón de eso, se desligaron o fueron excluidos de la institución.

\section{REFERENCIAS BIBLIOGRÁFICAS}

BRASIL (1996). M inistério da Saúde, Resolução no 196 de 10 de outubro de 1996: Diretrizes e normas regulamentadoras de pesquisa em seres humanos. O Mundo da Saúde, 21(1), 52-61.

BRASIL (2000). Portaria № 569, de 10 de junho 2000. Cria o Programa de Humanização ao Pré natal e Nascimento no âmbito do SistemaÚ nico de saúde. Braśli ia. Diário Oficial da União.

BRASI L (2001). M inistério da Saúde. Parto, Aborto e Puerpério. Assistência Humanizada à Mulher. Braślia. MS.

BROW N, A. (1982). Fathers' in the labour ward: medical and lay accounts. In: Mc Kee, L.O., Brien, M. (Eds). The father figure. New York. Tavistock Publications.

EN KIN , M. , LU M LEY, J., KEIRSE, M . J. (1995). Social and professional support during childbirth. In: Effective care in pregnancy and childbirth. New York. Oxford University Press.

ENKIN, M., Keirse, M.J.N.C., Renfrew, M . (1989). A guideto effectivecare in pregnancy and childbirth. Oxford. Oxford University Press.

EVAN S, R. (1999). Thepedagogic principal. Edmonton, Qual Institute Press.

FABRE, Z.L. (1992). Humanização em UTI pediátrica a equipe e a família. Arquivos Brasileiros de M edicina. 21,(3), 34-37.

FUSTIN ONI, S.M . (2000). As necessidades de cuidado da parturiente: uma perspectiva compreensiva da ação social. São Paulo. [Tese de doutorado - Escola de enfermagem da Universidade de São Paulo].

GUALDA, D.M.R. (1993). Eu conheço minha natureza: um estudo etnográfico da vivência do parto. [Tese de doutorado - Escola de enfermagem da Universidade de São Paulo].

HEM M IN K, E., VIRTA, A.L., KOPON EN , P. (1990). A trial on continuous human support during labour: feasibility, interventions and mother's satisfaction. Journal of psychosomatic Obstetrics and Gynecology; 11,(2), 239-250. 
HINDS, P.S.; CH AVES, D.E., CYPESS, S.M . (1992). Context as a source of meaning and understanding. In J.M. M orse (Ed.). Qualitative health research (pp. 31-42). Newbury Park, Sage.

HOFM ERYR, G.J., NIKODEM ,V.C., WOLM AN, W.L., Chalmers, B.E., Kramer, T. (1991). Companionship to modify the clinical birth environment: effects on progress and perception of labour and breast feeding. Journal Obstetric Gynecological. 98 (2), 756-64.

JANESICK, V.J. (1998). The dance of qualitative research design: methaphor, methodolatry, and meaning. In: Denzin, N.K., Lincoln, Y.S. (Eds). Strategies of qualitativeinquiry. (pp. 35-85). Thousand Oaks, Sage.

JENERAL, R.B.R. (2000). Vivendo um futuro incerto: a vivência da gravidez em uma comunidade. São Paulo. [Dissertação de mestrado - Escola de enfermagem da Universidade de São Paulo].

KLAUS, M .H., KEN NEL, J.H . (1993). Pais e bebes: a formação do apego. Porto Alegre, Artes M édicas.

KVALE, S. (1996). Interviews: an introduction to qualitative research interviewing. Thousand Oaks, Sage.

MEIHY, J.C.D.B. (1996). M anual de história oral. São Paulo, Loyola.

M INAYO, M .C.S. (1997). Ciência, técnica e arte: 0 desafio da pesquisa social. In: M inayo, M.C.S. et al. Pesquisa social: teoria, método e criatividade. Petrópolis, Vozes.
M ORSE, J.M . (1998). Designing funded qualitative research. In: Denzin, N.K., Lincoln, Y.S. (Eds). Strategies of qualitative inquiry (pp. 56-85). Thousand Oaks, Sage.

OCHIAI, A.M. (2000). O banho de chuveiro como medida de alívio da dor no trabalho de parto. São Paulo. [Dissertação de mestrado - Escola de enfermagem da Universidade de São Paulo.

ORGANIZAÇÃO MUNDIAL DA SAÚDE (1996). Assistência ao Parto Normal: um guia prático. Genebra: OMS.

POLIT, D.F., HUN GLER, B.P. (1995). Fundamentos de pesquisa em enfermagem. Porto Alegre, Artes M é dicas.

SÃO PAULO (ESTADO). (1999). Projeto de lei n. 10.241, de 17 de março de 1999. Dispõe sobre os direitos dos usuários dos serviços e das ações de saúde no Estado e dá outras providências (pp. 109:1). São Paulo, Diário Oficial do Estado.

SI LVA, W.V. (1999). Convivendo com obstáculos e fazendo o que se pode: a comunicação interpessoal na assistência pré-natal. [Tese de doutorado - Escola de enfermagem da Universidade de São Paulo].

VEH VILÃINEM-JULKUNEN, K., LINKOONEN, A. (1998). Father's experiences of childbirth. Midwifery. 14, (1), 10-17.

ZAGONEL, I.P.S. (1997). Contribuição do cuidado de enfermagem à humanização da parturição. Cogitare Enfermagem. 2 (1), 34-8. 